\title{
Analytical approximate solutions for investigating non-damped system of cantilever beams
}

\author{
Mirshaban Jafari ${ }^{2}$, Alireza Ahmadi ${ }^{1}$, Mehran Ghasempour ${ }^{1}$, Davood Domiri Ganji ${ }^{4}$, Ehsan Marzban ${ }^{4}$, Shahab Jafari ${ }^{4}$ \\ and Jamal Maktoubian ${ }^{3}$ \\ ${ }^{1}$ Department of Mechanical Engineering, Sari Branch, The university of Sari, Iran \\ ${ }^{2}$ University of applied science and technology, Department of Mechanical Engineering, Birjand University of Technology, Birjand, Iran \\ ${ }^{3}$ International School of Information Management(ISIM), University of Mysore, India \\ ${ }^{4}$ Department of Mechanical Engineering, Babol University of Technology, Babol, Iran
}

Received: 18 October 2016, Accepted: 20 April 2017

Published online: 7 February 2018.

\begin{abstract}
In the current study, a comprehensive analysis of nonlinear free vibrations of a flexible rotating beam attached with a setting angle to a rigid hub is perused. The system is supposed to be subjected to an external torque and a rotation dissipation load which collectively referred to as perturbations. A set of two strongly coupled second-order ordinary nonlinear differential equations with an ignorable coordinate is used to depict the dynamics of the individual mode in plane flexural deflection of the arm and its overall rotational motion. For the unperturbed system which is our especial case in this work, the system angular momentum parameter, not the hub rotational speed, becomes a specified constant of motion. Furthermore, in order to consider the effect of beam deflections on the overall motion and vice versa, utilizing torque-controlled approach is necessary. It is noteworthy that here three powerful analytical methods for instance Modified Differential Transformation Method (MDTM), Variation Iteration Method (VIM) and finally Homotopy Perturbation Method (HPM) have been introduced to investigate the comportment and frequency of cantilever rotating beams with a setting angle. These methods are useful and practical for solving a wide variety of nonlinear equations. Comparisons amongst the obtained results by all the presented methods and Numerical solution reveal that all the afore-mentioned methods are significantly effective and efficient especially MDTM.
\end{abstract}

Keywords: Cantilever beam, natural frequency, modified differential transformation method (MDTM).

\section{Introduction}

Nowadays, the rotating components are significantly noticed in a wide variety of industries such as turbine, compressors, helicopter rotor blades, airplane propellers, satellite antennas, robotic arms and various cooling fans. It is putative that the dynamic property of the above instruments has to be first thoroughly solved and understood if their high performance is perfectly fulfilled. The dynamic behavior of rotating beams is highly complex and thus in order to have an acceptable concept of this issue, they are generally modeled in the form of a simple rotating beam. Studies demonstrate that increasing the rotational speed tends to stiffen the beam (due to the centrifugal force) and thus increases its flexural natural frequencies and the beam deflections affect the overall motion of the rotating beam. Most of the studies like Hamdan and Al-Bedoor[1] considered the free vibrations of rotating beams (undamped system with no torque). In other words, the rotational speed was assigned a constant value or a time dependent function and introduced as an input to the system. Therefore in those studies, researchers did not consider the influence of the beam elastic vibrations on the rigid body motion. On the other hand, only few studies have looked after the more realistic, so called rigid-flexible coupling case where the rigid body motion is affected by the flexible beam elastic deflections in addition to the overall motion effect on these deflections. In a rotating undamped system, the magnitude and duration of the external torque which were applied for a specified starting period sets the steady state rotational speed or angular position.

The scrutiny for vibration characteristics of rotating beams has been investigated for a long period of time. A magnificent 


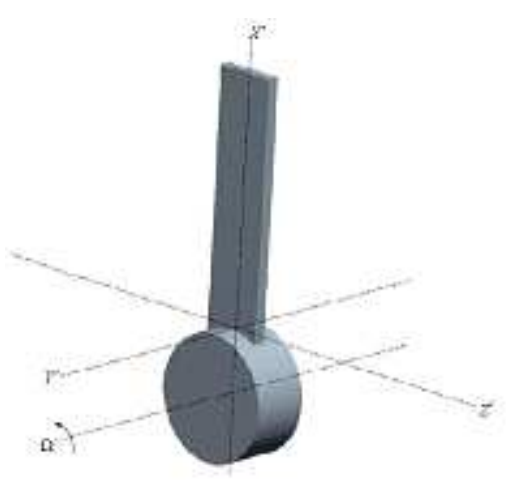

Fig. 1: The rotating straight beam.

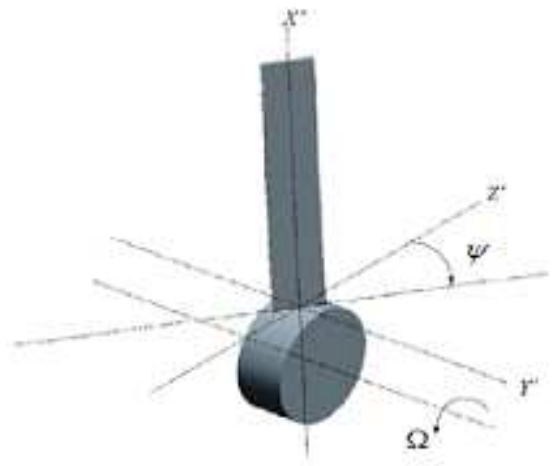

Fig. 2: The rotating beam with a setting angle $(\psi)$.

review of this issue can be found in published papers given by Leissa[2], Rammurati[3] and Rao[4]. Totally, the researches about the rotating beam from the orientation of the beam relative to the hub can be divided into the following several forms: I) the rotating straight beam (Fig.1). II) the rotating beam with a setting angle( $\psi)$ (Fig.2). III) the rotating beam with an inclined angle $(\eta u p)$ (Fig.3) and some other forms which are graphically described in the following chapter of this article in Fig.4 and Fig.5. Undoubtedly obtaining the exact solution of differential equations with variable coefficients for these kinds of system is not convenient but after some assumptions and elimination of parameters and a little simplification, we can extract the final governing equation for this mentioned system. Therefore, it will be very simple to evaluate natural frequency of the system.

Entirely, the basic solutions for rotating beam mechanism are often solved by approximation methods, take for example: the finite element method[5,6], the Galerkin method[7], the Rayleigh-Rits method[8], the perturbation method[9], the finite difference method[10] and the dynamic stiffness method[11]. But in this case study, Modified Differential Transformation Method (MDTM), Variation Iteration Method (VIM) and finally Homotopy Perturbation Method(HPM) have been utilized to investigate the mentioned system completely and to yield natural frequency of the proposed beam.

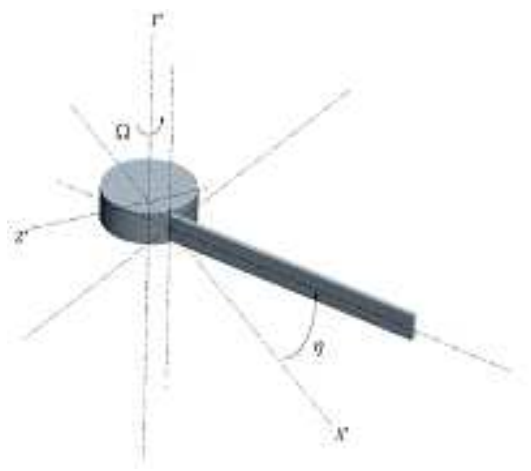

Fig. 3: The rotating beam with an inclined angle $(\eta u p)$.

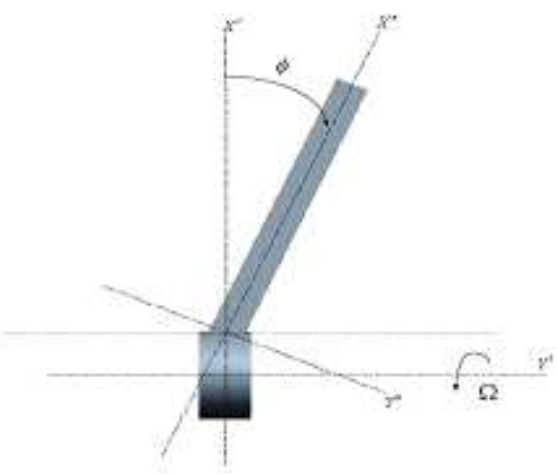

Fig. 4: The rotating beam with a flapping angle $(\Phi)$. 


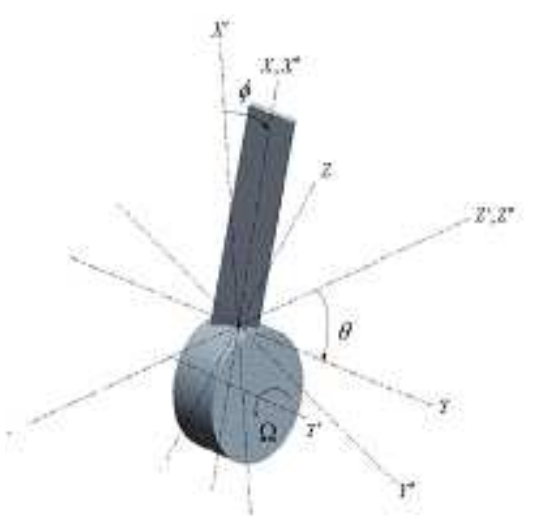

Fig. 5: The rotating beam with a flapping angle $(\Phi)$ and a setting angle $(\theta u p)$.

It is citable that in this case study, we mainly aim at the vibrational characteristics of rotating beams with a setting angle.

\section{Nomenclature}

\begin{tabular}{|c|c|}
\hline$R_{H}$ & Hub radius \\
\hline$m_{H}$ & Hub mass \\
\hline$\dot{\theta}$ & The angular velocity of hub \\
\hline$\psi$ & Setting angle \\
\hline$A_{b}$ & Uniform cross section area of the beam \\
\hline$E I$ & Flexural rigidity \\
\hline$L$ & Constant length of the beam \\
\hline$m_{b}$ & Beam mass \\
\hline$\rho$ & Beam mass density \\
\hline$C_{d R}$ & Assumed rotational linear viscous damping coefficient \\
\hline$\overline{C_{d T}}$ & Assumed bending linear viscous damping coefficient \\
\hline$\phi$ & Assumed mode shape \\
\hline$\varepsilon$ & Small positive parameter \\
\hline $\begin{array}{l}C_{0}=C+\frac{1}{3}+ \\
C^{2}\left(1+\frac{\mu}{2}\right)\end{array}$ & Dimensionless system parameter \\
\hline$\mu=m_{H} / m_{b}$ & Dimensionless system parameter \\
\hline$C=R_{H} / L$ & Dimensionless system parameter \\
\hline$\beta=\left(\frac{E T}{m_{b} l^{3}}\right)^{1 / 2}$ & Dimensionless system parameter \\
\hline$\beta_{i}, i=1, \ldots, 9$ & Dimensionless constant coefficient associated with the assumed mode of vibration \\
\hline$H$ & Angular momentum \\
\hline$P P_{\theta}$ & $\begin{array}{l}\text { Angular momentum of the system about the hub rotation axis divided by a mass } \\
\text { momentum of inertia factor }\end{array}$ \\
\hline $\bar{C}$ & Dimensionless hub radius to beam length ratio \\
\hline$\mu$ & Dimensionless hub mass to beam mass ratio \\
\hline$T_{A}$ & Constant amplitude of starting period \\
\hline$\overline{t_{s}}$ & Time duration of starting period \\
\hline
\end{tabular}




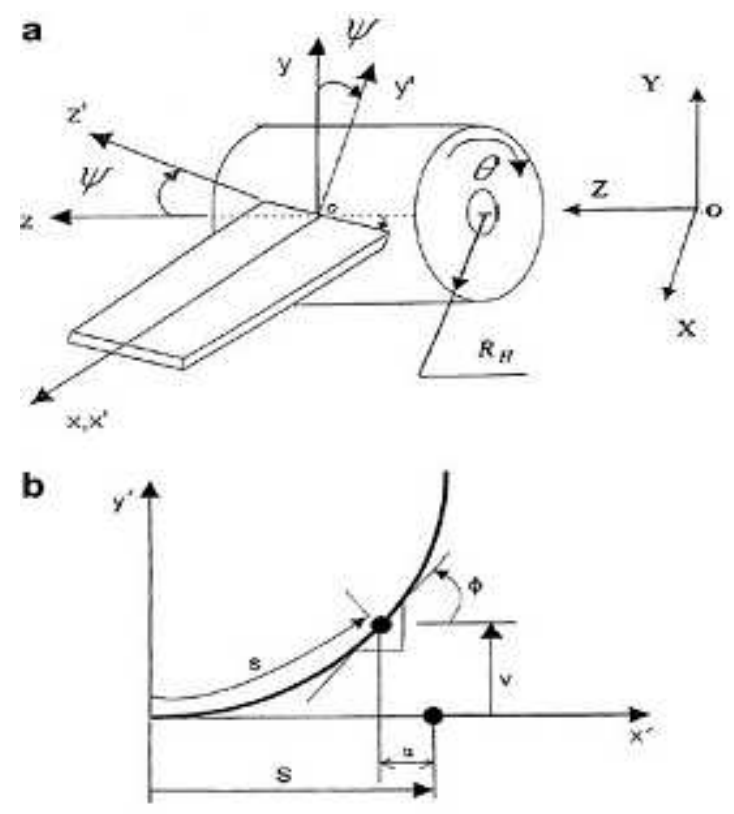

Fig. 6: (a). Cantilever beam diagram. (b). Deflected configuration of the arm.

\section{The dynamic model}

The cantilever beam which has been depicted in Fig.6 is attached to a hub which is assumed to be a rigid disk with radius $R_{H}, m_{H}$ and rotating at an angular velocity $\dot{\theta}$ about the $\mathrm{Z}$-axis. The effect of torque $\mathrm{T}$ on the hub causes it to rotate only. The X,Y,Z is system of fixed rectangular Cartesian coordinate axes with origin at the center of the hub. The $\mathrm{x}, \mathrm{y}, \mathrm{z}$ and the $x^{\prime}, y^{\prime}, z^{\prime}$ are two sets of rectangular Cartesian coordinate axes rotating with the hub with common origin at the root of the beam. The setting angle $\psi$ is rotation of the hub about longitude axis of the beam. The beam is assumed to be initially straight along the $x^{\prime}$-axis clamped at its base to the hub surface, having a uniform cross-sectional area $A_{b}$, flexural rigidity $E I$, constant length $l$, mass $m_{b}$ and density $\rho$. The beam thickness is assumed to be small compared to its length so that the effects of shear deformation and rotary inertia can be ignored. It is notable that various dynamic models have been proposed to represent the dynamics of rotating beams (e.g.[12-15]). In these models different approaches, leading to various results, are utilized to account for the axial displacement and centrifugal force. The mathematical model used here to describe the dynamics of the above beam system is a special case of the single mode model introduced in the work of Hamdan and El-Sinawi [16]. Therefore, the governing ordinary differential equations of the beam system are defined as follows.

$$
\begin{aligned}
& \left(\beta_{1}+\beta_{8} q^{2}\right) q^{*}+\left[\left(\beta_{7}-C \beta_{3}\right) \cos \psi+\frac{1}{2}\left(\beta_{5} \cos \psi\right) q^{2}\right] \theta \cdot\left[\beta^{2} \beta_{2}-\left(C \beta_{4}-\beta_{6}+\beta_{1} \cos ^{2} \psi\right) \dot{\theta}^{2}\right] q \\
& +\beta_{8} q \dot{q}^{2}+2 \beta^{2} \beta_{9} q^{3}+C_{d T} \dot{q}=0 \\
& \quad\left[C_{0}+\left(C \beta_{4}-\beta_{6}+\beta_{1} \cos ^{2} \psi\right) q^{2}\right] \ddot{\theta}+\cos \psi\left[\beta_{7}-C \beta_{3}+\frac{1}{2} \beta_{5} q^{2}\right] \ddot{q}+\left(\beta_{5} \cos \psi\right) q \dot{q}^{2} \\
& +2\left(C \beta_{4}-\beta_{6}-\beta_{1} \cos ^{2} \psi\right) q \dot{q} \dot{\theta}=\varepsilon\left(\frac{T}{m_{b} l^{2}}-C_{d R} \dot{\theta}\right)
\end{aligned}
$$

which

$$
\begin{aligned}
& \beta_{1}=\int_{0}^{1} \phi^{2} d \xi, \beta_{2}=\int_{0}^{1} \phi^{\prime \prime 2} d \xi, \beta_{3}=\int_{0}^{1} \phi d \xi, \beta_{4}=\int_{0}^{1}\left(\int_{0}^{\xi} \phi^{\prime 2} d \eta\right) d \xi, \beta_{5}=\int_{0}^{1} \phi\left(\int_{0}^{\xi} \phi^{\prime 2} d \eta\right) d \xi \\
& \beta_{6}=\int_{0}^{1} \xi\left(\int_{0}^{\xi} \phi^{\prime 2} d \eta\right) d \xi, \beta_{7}=\int_{0}^{1} \xi\left(\int_{0}^{\xi} \phi d \eta\right) d \xi \beta_{8}=\int_{0}^{1}\left(\int_{0}^{\xi} \phi^{\prime 2} d \eta\right)^{2} d \xi, \beta_{9}=\int_{0}^{1} \phi^{\prime 2} \phi^{\prime \prime 2} d \xi
\end{aligned}
$$


where, prime denotes a derivative with respect to the dimensionless arc length variable $\xi=\frac{s}{l}$. Note that the case $=0$ is used to indicate that the net driving torque, take for example the bracketed term in the right hand side of Eq. 2, is zero and will be referred to as the unperturbed system while the case $\neq 0$ (net driving torque not equal to zero) will be referred to as the perturbed system.

The degree of freedom in this dynamical system is the dimensionless modal beam tip lateral flexural deflection $q=\frac{v}{l}$ (see Fig.6) and the overall rigid body rotation $\theta u p$ which is an ignorable coordinate.

However, solving Eq.1 and Eq.2 analytically is more difficult since they have to be solved in two stages. In the first stage, one has a starting torque. The results of this step should be taken and substituted as initial conditions to the second stage in which one has a zero or a different specified torque. To facilitate analytical analysis of the governing model in Eq.1 and Eq. 2 for such systems, a coordinate transformation, which takes advantage of the fact that $\theta$ is an ignorable coordinate, is usually used to replace $\dot{\theta}$ by an angular momentum variable of the system[17].

First by defining the following constants.

$$
\begin{aligned}
& K_{1}=\beta_{1}, \quad K_{2}=\beta_{8}, \quad K_{3}=\left(\beta_{7}-C \beta_{3}\right) \cos \psi, \quad K_{4}=\frac{1}{2} \beta_{5} \cos \psi, \quad K_{5}=\beta^{2} \beta_{5} \\
& K_{6}=C \beta_{4}-\beta_{6}+\beta_{1} \cos ^{2} \psi, \quad K_{7}=2 \beta^{2} \beta_{9}
\end{aligned}
$$

Therefore, we can write Eq.1 and Eq.2 more briefly

$$
\begin{aligned}
& \left(K_{1}+K_{2} q^{2}\right) \ddot{q}+\left(K_{3}+K_{4} q^{2}\right) \ddot{\theta}+\left(K_{5}-K_{6} \dot{\theta}^{2}\right) q+K_{2} q \dot{q}^{2}+K_{7} q^{3}+C_{d T} \dot{q}=0 \\
& \left.c_{0}+k_{6} q^{2}\right) \ddot{\theta}+\left(k_{3}+k_{4} q^{2}\right) \ddot{q}+2 k_{4} q \dot{q}(\dot{q})^{2}+2 k_{6} q \dot{q} \dot{\theta}=\varepsilon\left(\frac{T}{m_{b} l^{2}}-C_{d R} \dot{\theta}\right)
\end{aligned}
$$

After the following substitution

$$
B=C_{0}+K_{6} q^{2} \quad \text { and } \quad D=K_{3}+K_{4} q^{2}
$$

into Eq.4, the above dynamic model becomes

$$
\begin{aligned}
& \left(K_{1}+K_{2} q^{2}\right) \ddot{q}+\left(K_{3}+K_{4} q^{2}\right) \ddot{\theta}+\left(K_{5}-K_{6} \dot{\theta}^{2}\right) q+K_{2} q \dot{q}^{2}+K_{7} q^{3}+C_{d T} \dot{q}=0 \\
& \frac{d}{d t}(B \dot{\theta}+D \dot{q})=\varepsilon\left(\frac{T}{m_{b} l^{2}}-C_{d R} \dot{\theta}\right)
\end{aligned}
$$

where B and D are given in Eq. 5. Moreover, if

$$
P_{\theta}=B \dot{\theta}+D \dot{q}
$$

Eq.(7) becomes

$$
\frac{d}{d t}\left(P_{\theta}\right)=\varepsilon\left(\frac{T}{m_{b} l^{2}}-C_{d R}\left(\frac{P_{\theta}-D \dot{q}}{B}\right)\right) .
$$

Note that $T=\frac{d H}{d t}$.

Next a coordinate transformation from the $\dot{?}$ and q coordinates to $P_{\theta}$ and $q$ coordinates is carried out by substituting

$$
\dot{\theta}=\frac{P_{\theta}-D \dot{q}}{B}
$$


In Eq.6 and Eq.7, performing the long mathematical manipulations and retaining up to third-order terms, the transformed equations are

$$
\begin{aligned}
& \left(S_{0}+S_{1} q^{2}\right) \ddot{q}+S_{4} q \dot{q}^{2}+\left(S_{7}-S_{8} P_{\theta}^{2}\right) q+S_{9} \dot{P}_{\theta} q^{2}+S_{10} q^{3}+S_{14} \dot{P}_{\theta}+C_{d T}\left(S_{15}+S_{16} q^{2}\right) \dot{q}=0 . \\
& \dot{P}_{\theta}=\varepsilon\left(\frac{T}{m_{b} l^{2}}-C_{d R}\left(\frac{P_{\theta}-\left(K_{3}+K_{4} q^{2}\right) \dot{q}}{\left(C_{0}+K_{6} q^{2}\right)}\right)\right)
\end{aligned}
$$

where

$$
\begin{aligned}
& S_{0}=C_{0}^{2} K_{1}-K_{3}^{2} C_{0}, S_{1}=2 K_{1} K_{6} C_{0}+C_{0}^{2} K_{2}-K_{3}^{2} K_{6}-2 K_{3} K_{4} C_{0}, \\
& S_{4}=K_{3}^{2} K_{6}-2 C_{0} K_{3} K_{4}+K_{2} C_{0}^{2}, S_{7}=K_{5} C_{0}^{2}, S_{8}=K_{6}, S_{9}=C_{0} K_{4}+K_{3} K_{6}, \\
& S_{10}=K_{7} C_{0}^{2}+2 K_{5} K_{6} C_{0}, S_{14}=C_{0} K_{3}, S_{15}=C_{0}^{2}, S_{16}=2 K_{6} C_{0} .
\end{aligned}
$$

It is noteworthy that $S_{0}, S_{1}, \ldots, S_{16}$ depend on the assumed mode of vibration and the system parameters $C, \Psi$ and $\mu$.

\section{Unperturbed system}

In this paper, what we mean by the unperturbed motion of the system is that system is assumed to rotate freely with zero net driving torque (right hand side of Eq.(12) is zero). In the present work, for simplicity of analysis, we take the rotational dissipation $C_{d R}$, as well as the bending damping $C_{d T}$ to be zero and the external applied torque $T$ to be the smooth half-sine-wave.

$$
\mathrm{T}=\left\{\begin{array}{l}
T_{A} \sin \left(\frac{\pi t}{t_{s}}\right) \text { if } 0 \leq t \leq t_{s} \\
0 \text { if } t \geq t_{s}
\end{array}\right\}
$$

Substituting for $C_{d R}=0$ and Eq. 14 into Eq. 12 and integrating the resulting equation from 0 to $t_{s}$ leads to the following expression for the unperturbed motion angular momentum parameter $P_{\theta}$.

$$
P_{\theta}=\frac{2 T_{A} t_{s}}{\pi m_{b} l^{2}}=\text { Constant }
$$

with units $\mathrm{S}^{-1}$. Thus $\dot{P}_{\theta}=0$ and Eq. (11) after setting $\mathrm{C}_{\mathrm{dT}}=0$, dividing by $\mathrm{S}_{0}$ and rearranging becomes

$$
\ddot{q}+\left(\frac{S_{7}}{S_{0}}-\frac{S_{8}}{S_{0}} P_{\theta}^{2}\right) q+\frac{S_{1}}{S_{0}} q^{2} \ddot{q}+\frac{S_{4}}{S_{0}} q \dot{q}^{2}+\frac{S_{10}}{S_{0}} q^{3}=0 .
$$

As expressed in the later chapter, the interest in this paper is with that the first mode of vibration which is supposed to dominate the dynamics of the rotating beam. So, the constant coefficients $\left(S_{0}, S_{1}, S_{4}, S_{7}, S_{8}, S_{10}\right)$ are evaluated based on this first mode vibration assumption.

In order to lighten the mathematical computation, we can rewrite Eq.16 with new definition for the constants as follows.

$$
\ddot{q}+\omega_{1}^{2} q+d_{1} q^{2} \ddot{q}+d_{2} q \dot{q}^{2}+d_{3} q^{3}=0
$$

where

$$
\omega_{1}^{2}=\frac{S_{7}}{S_{0}}-\frac{S_{8}}{S_{0}} P_{\theta}^{2} d_{1}=\frac{S_{1}}{S_{0}} d_{2}=\frac{S_{4}}{S_{0}} d_{3}=\frac{S_{10}}{S_{0}} .
$$

An approximate solution of Eq.1 is in the form of

$$
q(t)=A_{1} \cos \omega t+A_{3} \cos 3 \omega t
$$


and the initial conditions take the form of

$$
q(0)=A \dot{q}(0)=0
$$

It is noteworthy that there are two different modes of vibration according to table 1 as follows

Table 1: Values of variables in Eq. for the two modes.

\begin{tabular}{|l|l|l|l|}
\hline Mode & $\omega_{1}$ & $d_{1}$ & $d_{2}$ \\
\hline 1 & 1 & 1 & 1 \\
\hline 2 & 0 & 1 & 1 \\
\hline
\end{tabular}

As it was previously noted, in this case study we just analyze the first mode of vibration and for simplicity, we change the appearance of the first mode of vibration into the following form

$$
\frac{d^{2}}{d t^{2}} w(t)+w(t)+w(t)^{2}\left(\frac{d^{2}}{d t^{2}} w(t)\right)+w(t)\left(\frac{d}{d t} w(t)\right)^{2}+w(t)^{3}=0 .
$$

Also, we have changed the appearance of the boundary conditions in the form of

$$
W(0)=A=1, D(w(0))=0 .
$$

\section{Basic ideas}

\subsection{The basic idea of homotopy perturbation method}

Consider the following equation

$$
A(u)-f(r)=0, r
$$

With the boundary condition of

$$
B\left(u, \frac{\partial u}{\partial n}\right)=0 ; r \in \Gamma
$$

where $A$ is a general differential operator, $B$ denotes to the boundary operator, $f(r)$ is a given analytical function, $\Gamma$ is the boundary of domain $\Omega$ and $\frac{\partial u}{\partial n}$ refers to the differentiation along the normal drawn outwards from $\Omega$. The operator A can be divided into two parts: a linear part $L$ and a nonlinear part N [18-19]. Therefore, Eq.23 can be rewritten as follows

$$
L(u)+N(u)-f(r)=0 .
$$

In the case that the nonlinear Eq.23 has no small parameter, we can construct the following Homotopy

$$
H(v, p)=(1-p)\left[L(v)-L\left(u_{0}\right)\right]+p[A(v)-f(r)]=0
$$

where,

$$
v(r, p): \Omega \times[0,1] \rightarrow R
$$

In Eq.26, $p \in[0,1]$ is an embedding parameter and $u_{0}$ is the first approximation that satisfies the boundary condition. We can assume that the solution of considered equation can be obtained as a power series in $\mathrm{p}$, in this manner

$$
v=\sum_{i=0}^{n} p^{i} v_{i}=v_{0}+p v_{1}+p^{2} v_{2}+p^{3} v_{3}+\ldots
$$


And the best approximation for solution is

$$
u=\lim _{p \rightarrow 1} v=v_{0}+v_{1}+v_{2}+v_{3}+v_{4}+\ldots
$$

When Eq.26 corresponds to Eq.23 and Eq.29 becomes the approximate solution of the considered nonlinear equation. By increasing the number of series terms in Eq.28, the accuracy of the results will be augmented and they could be used as an exact solution.

\subsection{The basic idea of differential transformation method}

If $f(t)$ is analytic in the time domain $T$, then it will be differentiated continuously with respect to time $t$

$$
\varphi(t, k)=\frac{\left(d^{k} f(t)\right)}{d t^{k}}, \forall_{t}=T
$$

For $t=t_{i}$ then $\varphi(t, k)$, where $\mathrm{k}$ belongs to the set of non-negative integers, denoted as the K-domain. Therefore, Eq.30 can be rewritten as follows

$$
F(k)=\varphi\left(t_{i}, k\right)=\left[\frac{d^{k} f(t)}{d t^{k}}\right]_{t=t_{i}}, \forall_{t}=T
$$

where $\mathrm{F}(\mathrm{k})$ is called the spectrum of $\mathrm{f}(\mathrm{t})$ at $t=t_{i}$ in the K-domain. If $f(t)$ can be represented by the Taylor series, then it can be represented as

$$
f(t)=\sum_{k=0}^{\infty}\left(\frac{\left(t-t_{i}\right)^{k}}{k !}\right) \cdot F(k) .
$$

Eq.32 is called the inverse transform of $F(k)$. Then, according to the symbol D which is denoting the differential transform process and its combination by Eq.31 and Eq.32, we obtain

$$
f(t)=\sum_{k=0}^{\infty}\left(\frac{\left(t-t_{i}\right)^{k}}{k !}\right) \cdot F(k) \equiv D^{-1} F(k) .
$$

From the above definitions, it is easy to obtain the basic mathematical operations of DTM in Table 2 as

Table 2: Basic transformation for simple functions by DTM.

\begin{tabular}{|l|l|}
\hline Time definition & Transformed function \\
\hline$w(t)=\alpha \cdot u(t) \pm \beta \cdot v(t)$ & $W(k)=\alpha \cdot U(k) \pm \beta \cdot V(k)$ \\
\hline$w(t)=\left(d^{m} u(t)\right) /\left(d t^{m}\right)$ & $\mathrm{w}(\mathrm{k})=\frac{(\mathrm{k}+\mathrm{m}) !}{\mathrm{k} !} U(k+m)$ \\
\hline$W(t)=u(t) v(t)$ & $W(k)=\sum_{l=0}^{k} U(l) \cdot V(k-l)$ \\
\hline$w(t)=t^{m}$ & $W(k)=\delta(k-m)= \begin{cases}1 & \text { if } k=m \\
0 & \text { if } k \neq m \\
& k\end{cases}$ \\
\hline$w(t)=\exp (t)$ & $W(k)=\frac{1}{k !}$ \\
\hline$w(t)=\sin (\omega t+\alpha)$ & $W(k)=\left(\frac{\omega^{k}}{k !}\right) \sin \left(\frac{k \pi}{2}+\alpha\right)$ \\
\hline$W(t)=\cos (\omega t+\alpha)$ & $W(k)=\left(\frac{\omega^{k}}{k !}\right) \cos \left(\frac{k \pi}{2}+\alpha\right)$ \\
\hline
\end{tabular}


To end up, using the differential transform [20], a differential equation in the domain of interest can be transformed into an algebraic equation in the $\mathrm{k}$ domain and $\mathrm{f}(\mathrm{t})$ can be obtained by the finite-term Taylor series expansion plus a remainder as

$$
f(t)=\sum_{k=0}^{\infty}\left(\frac{\left(t-t_{i}\right)^{k}}{k !}\right) \cdot F(k)+R_{N+1}(t)
$$

\subsection{Variation iteration method}

To clarify the basic ideas of VIM, we consider the following differential equation

$$
L u+F u=g(t)
$$

where $L$ is a linear operator, $F$ is a nonlinear operator and $g(t)$ is a heterogeneous term. According to VIM, we can write down a correction function as follows

$$
u_{n+1}(t)=u_{n}(t)+\int_{0}^{t} \lambda\left(L u_{n}(\tau)+F \tilde{u}_{n}(\tau)-g(\tau)\right) d \tau
$$

where $\lambda$ is a general Lagrangian multiplier which can be identified optimally via the variational theory. The subscript $n$ indicates the $n t h$ approximation and $u$ 'n is considered as a restricted variation [21-23], i.e., $\delta u n=0$.

\section{Applications}

\subsection{Homotopy perturbation method}

Considering Eq.1, according to the HPM, the homotopy, Eq.35 is gained as follows

$$
\begin{aligned}
H(f, p) & =(1-p)\left(\frac{d^{2}}{d t^{2}} w(t)+w(t)\right)+p\left(\frac{d^{2}}{d t^{2}} w(t)+w(t)+w(t)^{2}\left(\frac{d^{2}}{d t^{2}} w(t)\right)+w(t)\left(\frac{d}{d t} w(t)\right)^{2}+w(t)^{3}\right) H(f, p) \\
& =(1-p)\left(\frac{d^{2}}{d t^{2}} w(t)+w(t)\right)+p\left(\frac{d^{2}}{d t^{2}} w(t)+w(t)+w(t)^{2}\left(\frac{d^{2}}{d t^{2}} w(t)\right)+w(t)\left(\frac{d}{d t} w(t)\right)^{2}+w(t)^{3}\right) .
\end{aligned}
$$

On the basis of HPM theory, we assume $\mathrm{f}(\eta)$ in the form of

$$
\mathrm{w}(\mathrm{t})=\mathrm{w}_{0}(\mathrm{t})+\mathrm{pw}_{1}(\mathrm{t})+\mathrm{p}^{2} \mathrm{w}_{2}(\mathrm{t})+\mathrm{p}^{3} \mathrm{w}_{3}(\mathrm{t})+\ldots
$$

Now, after substituting Eq.38 into Eq.37 and some simplification and rearranging them which were based on powers of p-terms, we have

$$
\begin{gathered}
P^{0}: w_{0}(t)+\frac{d^{2}}{d \tau^{2}} w_{0}(t) \\
p^{1}: \frac{d^{2}}{d t^{2}} w_{1}(t)+w_{1}(t)+w_{0}(t)\left[\frac{d}{d t} w_{0}(t)\right]^{2}+w_{0}(t)^{2}\left[\frac{d^{2}}{d t^{2}} w_{0}(t)\right] \\
p^{2}: 3 w_{0}(t)^{2} w_{1}(t)+2 w_{0}(t)\left[\frac{d}{d t} w_{0}(t)\right]\left[\frac{d}{d t} w_{1}(t)\right]+\frac{d^{2}}{d t^{2}} w_{2}(t)+w_{0}(t)^{2}\left[\frac{d^{2}}{d t^{2}} w_{1}(t)\right] \\
+2 w_{0}(t) w_{1}(t)\left[\frac{d^{2}}{d t^{2}} w_{0}(t)\right]+w_{2}(t)+w_{1}(t)\left[\frac{d}{d t} w_{0}(t)\right]^{2}
\end{gathered}
$$




$$
\begin{aligned}
p^{3} & : w_{1}\left(t^{2}\right)\left[\frac{d^{2}}{d t^{2}} w_{0}(t)\right]+3 w_{0}(t)+w_{1}(t)^{2}+w_{2}(t)\left[\frac{d}{d t} w_{0}(t)\right]^{2}+3 w_{0}(t)^{2} w_{2}(t)+2 w_{0}(t) w_{2}(t)\left[\frac{d^{2}}{d t^{2}} w_{0}(t)\right] \\
& +2 w_{0}(t) w_{1}(t)\left[\frac{d^{2}}{d t^{2}} w_{1}(t)\right]+2 w_{0}(t)\left[\frac{d}{d t} w_{0}(t)\right]\left[\frac{d}{d t} w_{2}(t)\right]+w_{0}(t)^{2}\left[\frac{d^{2}}{d t^{2}} w_{2}(t)\right]+w_{3}(t)+\frac{d^{2}}{d t^{2}} w_{3}(t) \\
& +2 w_{1}(t)\left[\frac{d}{d t} w_{0}(t)\right]\left[\frac{d}{d t} w_{1}(t)\right]+w_{0}(t)\left[\frac{d}{d t} w_{1}(t)\right]
\end{aligned}
$$

By continuing the above process, higher accuracy will be obtained. Solving Eq.39 to Eq. 42 with considering appropriate initial conditions which means the right hand side of the initial conditions should be equal to zero that is $\left\{w(t=0)=0, w^{\prime}(t=0)=0\right\}$ for the afore-mentioned equations with the exception of Eq.39 which for this case we should consider the main boundary condition, Eq.22. Therefore, we will have

$$
\begin{aligned}
& w_{0}(t)=\cos (t) \\
& w_{1}(t)=-\frac{1}{8} \sin (t)(t-\cos (t) \sin (t)) \\
& w_{2}(t)=\frac{1}{256} \cos (t)+\frac{3}{64} \cos (t)^{2} \sin (t) t+\frac{9}{256} \sin (t) t-\frac{1}{128} \cos (t) t^{2}+\frac{5}{64} \cos (t)^{5}-\frac{21}{256} \cos (t)^{3}
\end{aligned}
$$

Therefore, in regard to the given explanations, $\mathrm{w}(\mathrm{t})$ will be achieved as follows

$$
\left\{\mathrm{w}(\mathrm{t})=\lim _{p \rightarrow 1} \mathrm{w}_{0}(\mathrm{t})+\mathrm{p} \cdot \mathrm{w}_{1}(\mathrm{t})+\mathrm{p}^{2} \cdot \mathrm{w}_{2}(\mathrm{t})+\mathrm{p}^{3} \cdot \mathrm{w}_{3}(\mathrm{t})+\ldots\right\}
$$

Eventually, we have

$$
\begin{aligned}
w(t)= & \frac{4345}{4096} \cos (t)-\frac{1}{8} \sin (t)(t-\cos (t) \sin (t))+\frac{15}{256} \cos (t)^{2} \sin (t) t+\frac{9}{256} \sin (t) t-\frac{1}{128} \cos (t) t^{2}+ \\
& \frac{941}{6144} \cos (t)^{5}-\frac{21}{256} \cos (t)^{3}-\frac{103}{1536} \cos (t)^{7}-\frac{25}{512} \cos (t)^{4} \sin (t) t+\frac{1}{12288}\left(40+108 t^{2}\right) \cos (t)^{3} \\
& +\frac{1}{12288}\left(-837-6 t^{2}\right) \cos (t)+\frac{1}{12288}\left(4 t^{3}-234 t\right) \sin (t) .
\end{aligned}
$$

As it is obvious, solution of terms varies periodically and in each step more accuracy has been gained. So, $\omega(t)$ can be charted in Fig.7 as follows

\subsection{Variational iteration method}

In order to solve Eq. 21 with the boundary conditions of Eq. 22 utilizing VIM, we construct a correction function as follows

$$
w_{n+1}(t)=w_{n}(t)+\int_{0}^{t} \lambda\left(\frac{d^{2}}{d \tau^{2}} w_{n}(\tau)+w_{n}(\tau)+w_{n}(\tau)^{2}\left(\frac{d^{2}}{d \tau^{2}} w_{n}(\tau)\right)+w_{n}(\tau)\left(\frac{d}{d \tau} w_{n}(\tau)\right)^{2}+w_{n}(\tau)^{3}\right) d \tau
$$

It is notable that recently a new method has been introduced in order to find lagrange multiplier which needs less computational work in comparison to the previous method as follows

To start, it is necessary to separate the linear part of the main differential equation which in this case study is Eq.(21), afterwards this is the best time to take its Laplace transform. For the next step, we should utilize the first term of Derivative Laplace Transform Theorem and equals it to $(-1)^{n}$ where $n$ denotes to the highest order of derivative of the linear part of our equation. Then with taking inverse Laplace transform and substitution (A-B) instead of the variant of our answer which A and B refer to the integral variant of our correction function and the top range of integral, respectively. It is easy to understand the afore-mentioned procedure in accordance to these formulae.

The Lagrangian multiplier can therefore be computed in the form of

(1) Separating the linear part of the equation $\Rightarrow \frac{d^{2}}{d t^{2}} w(t)+w(t)$ 


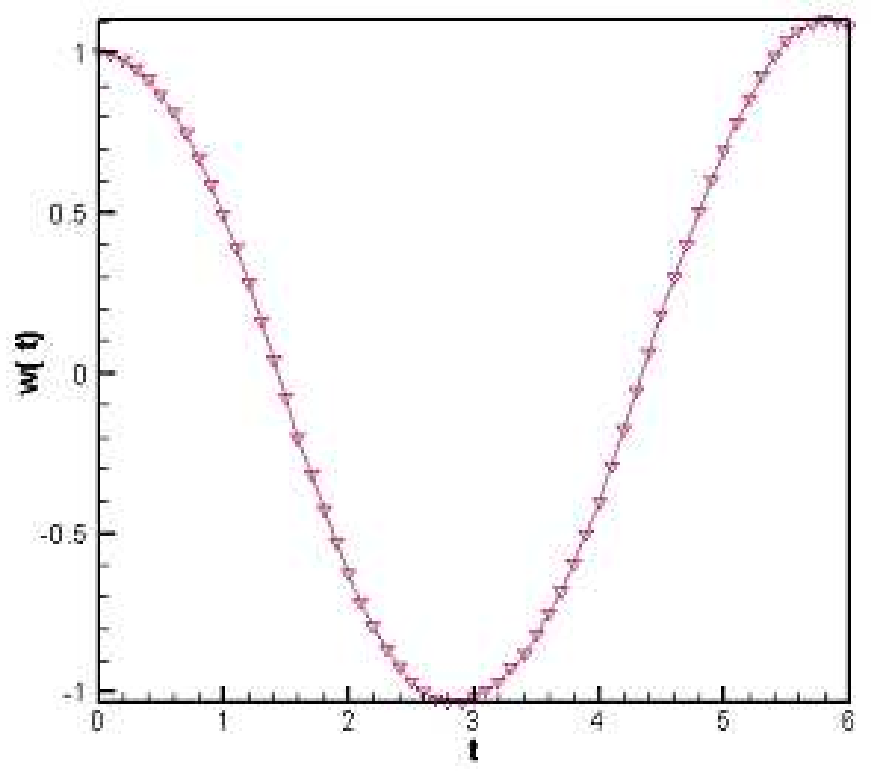

Fig. 7: The result of HPM for $\omega(t)$ in the time domain between $0 \leq t \leq 6$.

(2) Taking its Laplace transform $\Rightarrow s^{2} L\{w(t)\}+L\{w(t)\}=1$. So $L\{w(t)\}\left(s^{2}+1\right)=1$ then we have $L\{w(t)\}=\frac{1}{1+s^{2}}$ therefore $w(t)=L^{-1}\left\{\frac{1}{1+s^{2}}\right\}=\sin (t)$

(3) In as much as $\lambda=\lim _{t \rightarrow \tau-t} w(t)$, finally $\lambda$ is obtained in the following form

$$
\lambda=\sin (\tau-t)
$$

As a result, we obtain the following iteration formula

$$
w_{n+1}(t)=w_{n}(t)+\int_{0}^{t} \lambda\left(\frac{d^{2}}{d t^{2}} w_{n}(\tau)+w_{n}(\tau)+w_{n}(\tau)^{2}\left[\frac{d^{2}}{d t^{2}} w_{n}(\tau)\right]+w_{n}(\tau)\left[\frac{d}{d t} w_{n}(\tau)\right]^{2}+w_{n}(\tau)^{3}\right) d \tau .
$$

Now we start with an arbitrary initial approximation that satisfies the initial condition

$$
w_{0}(t)=A \cos (t)
$$

On the basis of Eq.50, we have

$$
w_{1}(t)=w_{0}(t)+\int_{0}^{t}\left(-\sin (-\tau+t)\left(\frac{d^{2}}{d t^{2}} w_{0}(\tau)+w_{0}(\tau)^{2}\left[\frac{d^{2}}{d t^{2}} w_{0}(\tau)\right]+w_{0}(\tau)\left[\frac{d}{d t} w_{n}(\tau)\right]^{2}+w_{0}(\tau)^{3}\right) d \tau .\right.
$$

Substituting Eq. (51) into Eq. (52) and after some simplification, we have

$$
w_{1}(t)=\frac{9}{8} \cos (t)-\frac{1}{8} t \sin (t)-\frac{1}{8} \cos (t)^{3}
$$

And so on.

In the same manner, the rest of the components of the iteration formula can be obtained. Therefore, the diagram of $\omega(t)$ is illustrated in Fig. 8 as 


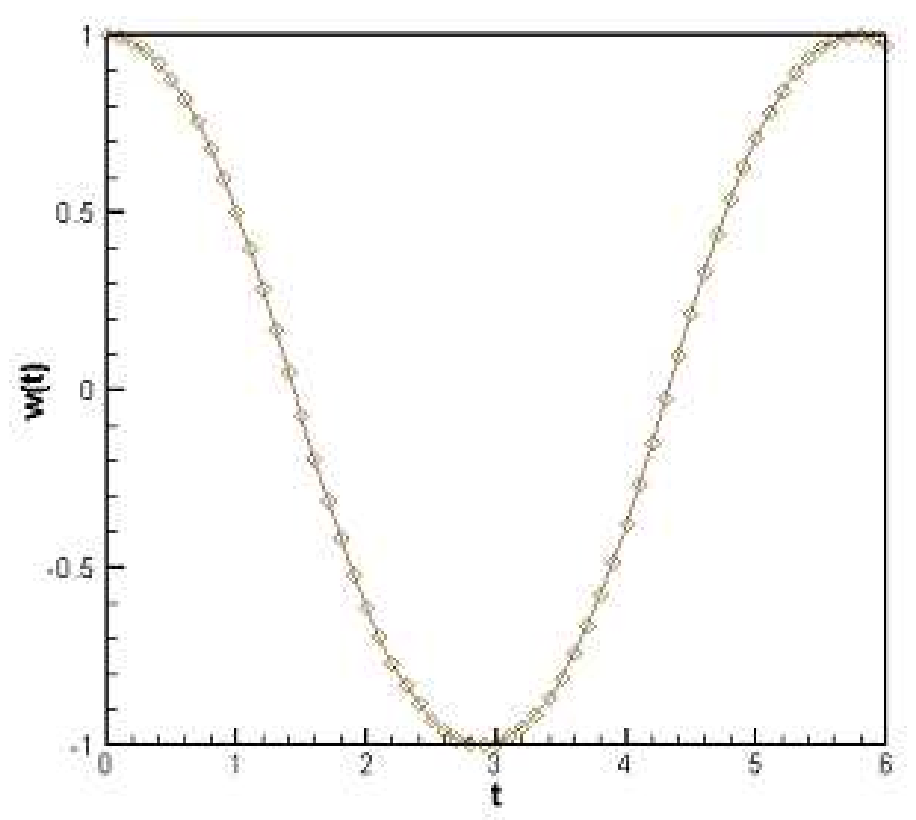

Fig. 8: The result of VIM for $\omega(t)$ in the time domain between $0 \leq t \leq 6$.

\subsection{Differential transformation method}

With regard to the explanation of differential transformation Method which was discussed completely in section 4.2, Eq.21 can be solved as follows

$$
\begin{aligned}
& (k+1)(k+2) W(k+2)+W(k)+\sum_{k_{1}=0}^{k} \sum_{L=0}^{k_{1}} W(L) W\left(k_{1}-L\right)\left(k+1-k_{1}\right)\left(k+2-k_{1}\right) W\left(k+2-k_{1}\right) \\
& \quad+\sum_{k_{1}=0}^{k} \sum_{L=0}^{k_{1}} W(L)\left(k+1-k_{1}\right)\left(k_{1}-L+1\right) W\left(k_{1}-L+1\right) W\left(k+1-k_{1}\right) \\
& \quad+\sum_{k_{1}=0}^{k} \sum_{L=0}^{k_{1}} W(L) W\left(k_{1}-L\right) W\left(k-k_{1}\right)=0 .
\end{aligned}
$$

Then, we should take differential transform from the boundary conditions in the form of $D\{w(t=0)\}=D\{1\}$ and $D\left\{\left.\frac{d}{d t} w(t)\right|_{t=0}\right\}=D\{0\}$ so, we have

$$
W(0)=1 \quad \text { and } \quad W(1)=0
$$

In this step, we dedicate some values to $\mathrm{K}$ which are from 2 to 6 (because according to the Eq.(55) we have the amounts of $W(k)$ for $k=0$ and 1$)$ then by using maple package, we obtain these equations

$$
W(2)=-\frac{1}{2}
$$

and

$$
W(3)=W(4)=W(5)=W(6)=W(7)=0
$$




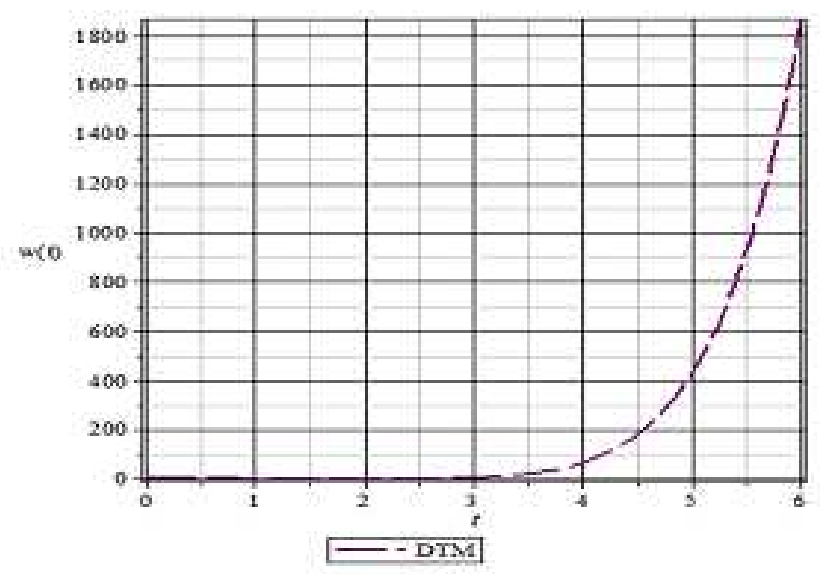

Fig. 9: The result of VIM for $\omega(t)$ in the time domain between $0 \leq t \leq 6$.

then

$$
W(8)==\frac{1}{896}
$$

With respect to the above equations, we can define $w(t)$ as follows:

$$
w(t)=\sum_{k=0}^{8} W(k) \cdot t^{k}
$$

Therefore, we have:

$$
w(t)=1-\frac{1}{2} t^{2}+\frac{1}{896} t^{8}
$$

Finally, the chart of the $w(t)$ by DTM is depicted in Fig.9 as follows. Obviously, DTM is not a suitable method to solve these kinds of equations. Therefore, another analytical approach is required to overcome this difficulty such as MDTM as follows

\subsection{Modified differential transformation method}

As regards Fig.9. DTM is not accurate for solving this problem so it should be improved by little changes in solution procedure which is named MDTM or MSDTM. Briefly, the story of MDTM or MSDTM is the same as DTM but here, the solution domain is detached into small pieces and boundary conditions are applied for each of these small pieces of domain then these proceedings together can prevent a sudden rise in the specified domain. With regard to the afore-mentioned explanations, the solution procedure is done as follows.

$$
w_{0}(t)=1-\frac{1}{2} t^{2}+\frac{1}{896} t^{8}
$$

and

For the next step

Afterthat

Then

$$
\begin{gathered}
w_{0.5}(t=0)=0.875004 \\
w_{1.0}(t=0)=0.501612 \\
w_{1.5}(t=0)=-0.07267
\end{gathered}
$$$$
w_{2.0}(t=0)=-0.61244
$$

and

and

and

and

$$
\begin{gathered}
w_{0.5}(t=1)=-0.499930 \\
w_{1.0}(t=1)=-0.986016 \\
w_{1.5}(t=1)=-1.22054
\end{gathered}
$$$$
w_{2.0}(t=1)=-0.872408
$$ 


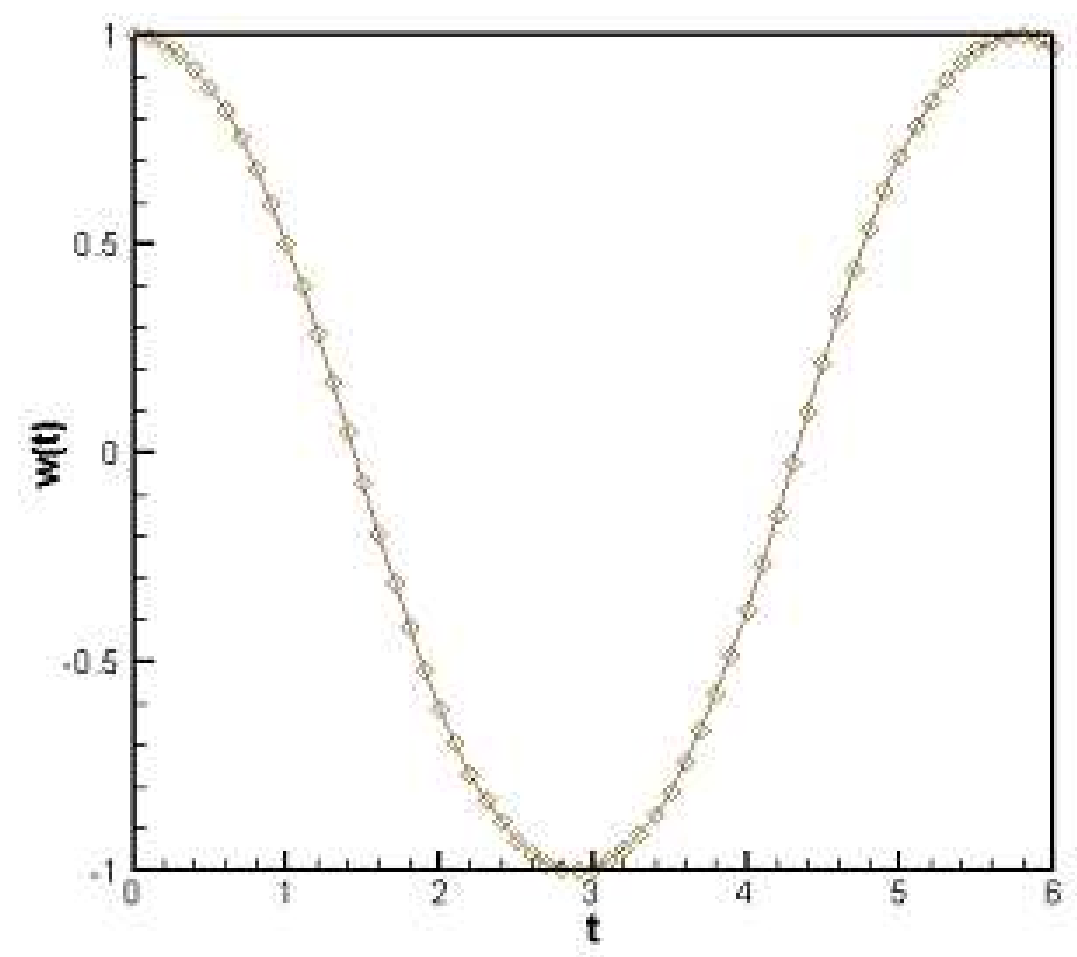

Fig. 10: The solution of Eq. 21 resulted by MDTM.

The other values are computed in the form of

$$
w_{2.5}(t=0)=-0.92594 \text { and } w_{2.5}(t=1)=-0.376913
$$

$\begin{array}{llll}\text { and } & w_{3.0}(t=0)=-0.98977 & \text { and } & \\ \text { So } & w_{3.5}(t=0)=-0.80438 & \text { and } & w_{3.0}(t=1)=0.12157 \\ \text { Afterwards } & w_{4.0}(t=0)=-0.37424 & \text { and } & w_{4.0}(t=1)=1.08339 \\ \text { Then } & w_{4.5}(t=0)=0.218356 & \text { and } & w_{4.5}(t=1)=1.18731 \\ \text { Forthenextstep } & w_{5.0}(t=0)=0.716692 & \text { and } & w_{5.0}(t=1)=0.784768 \\ \text { Then } & w_{5.5}(t=0)=0.981847 & \text { and } & w_{5.5}(t=1)=0.274777 \\ \text { Eventually } & w_{6.0}(t=0)=0.991848 & \text { and } & w_{6.0}(t=1)=-0.234724\end{array}$

Now, the solution procedure is done completely. Therefore, the diagram of $\omega(t)$ has been achieved in Fig.10 as

\subsection{Graphical results}

According to the obtained formulae from the previous parts of this paper, a comparison amongst the achieved solutions by HPM,VIM and MDTM is yielded in Fig.11 as follows Eventually, the error charts of the mentioned methods are illustrated in Fig. 12 as And also the above graphical results are shown in table 3 as follows 


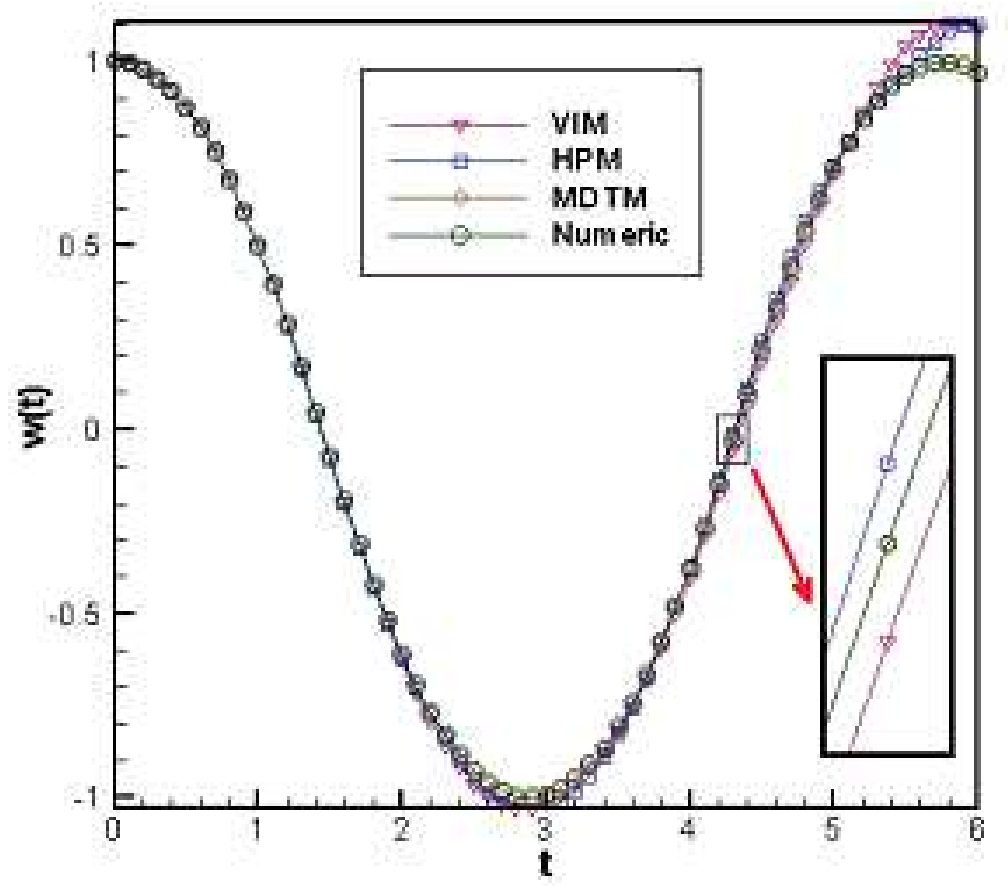

Fig. 11: A comparison amongst MDTM, VIM, HPM and numerical solution.

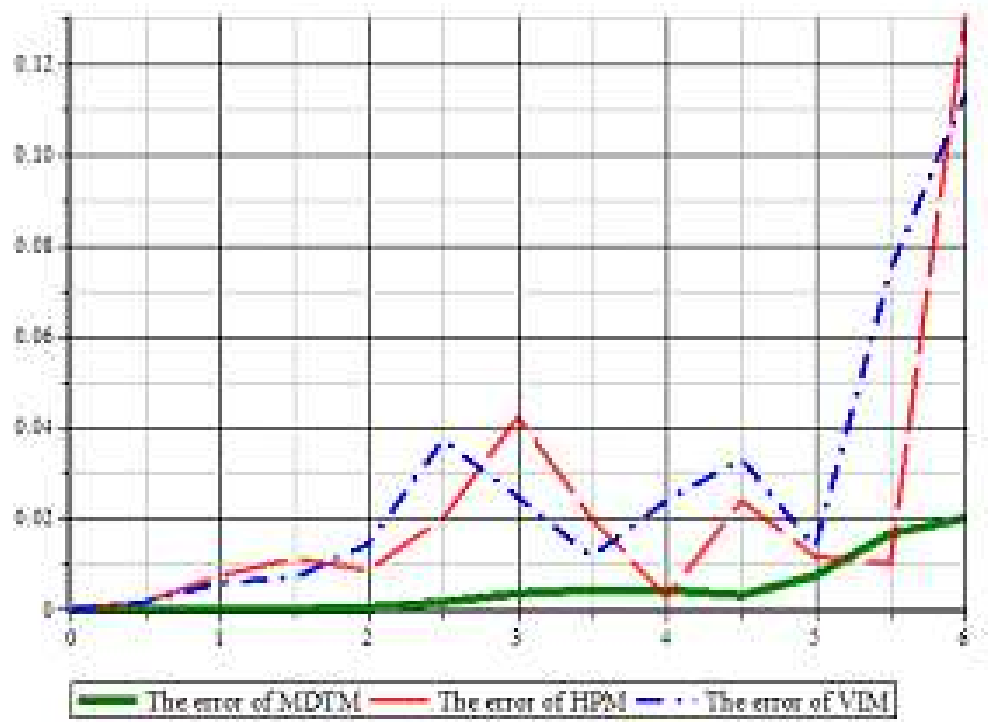

Fig. 12: The computational error of $w(t)$ in the specified domain resulted by HPM,VIM and MDTM. 
Table 3: Obtained numerical values and errors for $w(t)$ in the specified domain.

\begin{tabular}{|l|l|l|l|l|l|l|l|}
\hline $\mathbf{t}$ & Numeric & HPM & VIM & MDTM & $\begin{array}{l}\text { The } \\
\text { Error of } \\
\text { HPM }\end{array}$ & $\begin{array}{l}\text { The } \\
\text { Error of } \\
\text { VIM }\end{array}$ & $\begin{array}{l}\text { The } \\
\text { Error of } \\
\text { MDTM }\end{array}$ \\
\hline 0 & 1 & 1 & 1 & 1 & 0 & 0 & 0 \\
\hline 0.5 & 0.87500480 & 0.87342313 & 0.87344892 & 0.87500437 & 0.00158166 & 0.00155588 & 0.00000043 \\
\hline 1 & 0.50162102 & 0.49433444 & 0.49600557 & 0.50161223 & 0.00728657 & 0.00561544 & 0.00000875 \\
\hline 1.5 & 0.07260543 & 0.08371294 & 0.07936376 & 0.07267108 & 0.01110750 & 0.00675831 & 0.00006564 \\
\hline 2 & 0.61223450 & 0.62077682 & 0.62629234 & 0.61244206 & 0.00854232 & 0.01405784 & 0.00020755 \\
\hline 2.5 & 0.92732018 & 0.94736007 & 0.96436965 & 0.92594631 & 0.02003988 & 0.03704946 & 0.00137380 \\
\hline 3 & 0.99295063 & 1.03511220 & 1.01747393 & 0.98977396 & 0.04216160 & 0.02452329 & 0.00317666 \\
\hline 3.5 & 0.80860942 & 0.82864765 & 0.82013868 & 0.80438519 & 0.02003822 & 0.01152925 & 0.00422423 \\
\hline 4 & 0.37851282 & 0.37591470 & 0.40225586 & 0.37424330 & 0.00259811 & 0.02374304 & 0.00426952 \\
\hline 4.5 & 0.21532634 & 0.23896539 & 0.18224813 & 0.21835681 & 0.02363904 & 0.03307821 & 0.00303046 \\
\hline 5 & 0.70943825 & 0.72077476 & 0.69595767 & 0.71669247 & 0.01133650 & 0.01348058 & 0.00725421 \\
\hline 5.5 & 0.96554062 & 0.97549322 & 1.03982953 & 0.98184710 & 0.00995260 & 0.07428890 & 0.01630648 \\
\hline 6 & 0.97180256 & 1.10238841 & 1.08513912 & 0.99184812 & 0.13058584 & 0.11333656 & 0.02004556 \\
\hline
\end{tabular}

Due to Fig.12 and Table.3, the maximum errors of HPM, VIM and MDTM in the specified domain are approximately $\% 13, \% 11$ and $\mathbf{\% 2}$, respectively. Therefore, it is notable that amongst the three mentioned analytical methods, the achieved solution by MDTM is considered approximately as the real answer of the presented problem.

\section{Conclusions}

The research undertaken in this paper is devoted to the nonlinear vibrations of an inextensible rotating beam fixed with a setting angle to a rigid hub. Moreover, the free vibration of this rotating beam under a determined starting torque using the transformed equations has been investigated thoroughly. Therefore by assuming a prescribed starting torque instead of a prescribed overall motion, the beam deflection influence on the rigid body motion and vice versa which means the effect of rigid body motion on the beam deflection was acquired. Afterwards, three analytical methods were utilized in order to obtain the vibrational frequency of the mentioned beam and the outcomes have been compared with numerical results in order to check their precision and accuracy. It is noteworthy that the obtained results by MDTM, VIM and HPM have been showed graphically and compared together to get the most suitable and precise solution. Eventually, on the basis of the obtained results from Table.3 and Fig.12, it is clear that amongst all of the applied analytical methods, MDTM is very applicable in this special case.

\section{Competing interests}

The authors declare that they have no competing interests.

\section{Authors' contributions}

All authors have contributed to all parts of the article. All authors read and approved the final manuscript.

\section{References}

[1] M.N. Hamdan, B.O. Al-Bedoor, Non-linear free vibrations of a rotating flexible arm, Journal of Sound and Vibration 242, 83985,2001 .

[2] A. Leissa Vibrational aspects of rotating turbo machinery blades. ASME applied mechanics reviews vol 34. No pp 629-635,1981

[3] V. Ramamurti and P. Balasubramanian, Analysis of turbo machine blades: a review, the shock and vibration digest.Vol 16. No $8 \mathrm{pp}$ 13-28, 1984. 
[4] J.S. Rao "Turbomachine blade vibration." the shock and vibration digest" Vol 19 No 5. Pp 3-10, 1987.

[5] T Yokoyama, Free vibration characteristics of rotating Timoshenko beams, "International journal of mechanical science" Vol30. No 10. PP 743-755,1988.

[6] S. Mulmule G. Singh and GV. Rao "Flexural vibration of rotating tapered Timoshenko beams, Journal of sound and vibration. Vol 160. No 2. pp 372-377,1993.

[7] T.H. Young, T.M Lin " Stability of rotating pretwisted , tapered beams with randomly varing speeds, ASME Journal of vibration of vibration and Acoustics. Vol 120. Pp 784-790, 1998.

[8] H.H Yoo .S.H.Shin vibration analysis of rotating cantilever beams. Journal of sound and vibration, Vol 212, No 5. Pp 807-828, 1998.

[9] F.S.M. Jarrar, M.N Hamdan , Nonlinear vibrations and buckling of a flexible rotating beam, a prescribed torque approach, Mechanics and machine theory, Vol 42, pp 919-939, 2007.

[10] C.L.Ko, 'Dynamic analysis for free vibration of rotating sandwiches tapered beam, AIAA Journal Vol27. No 10, pp 1425-1433, 1989.

[11] J.R. Banerjee . H.Su. D.R.Jackson free vibration of rotating tapered beams using the dynamic stiffness method, Journal of sound and vibration. Vol 298. PP. 1034-1054, 2006.

[12] H.H. Yoo, R.R. Ryan, R.A. Scott, Dynamics of flexible beams undergoing overall motion, Journal of Sound and Vibration 181, 261-278,1995.

[13] S. Seo, H.H. Yoo, Dynamic analysis of flexible beams undergoing overall motion employing linear strain measures, AIAA Journal 40, 319-326,2002.

[14] J. Chung, H.H. Yoo, Dynamic analysis of a rotating cantilever beam by using the finite element method, Journal of Sound and Vibration 249, 147-164,2002.

[15] J. Chung, D. Jung, H.H. Yoo, Stability analysis for the flapwise motion of a cantilever beam with rotary oscillation, Journal of Sound and Vibration 273, 1047-1062, 2004.

[16] Hamdan, M.N and A.H. El-Sinawai, On the nonlinear vibration of an inextensible rotating arm with setting angle and flexible hub. J. Sound and vibration, 281:375-398, 2005.

[17] S.W. Shaw, Chaotic dynamics of a slender beam rotating about its longitudinal axis, Journal of Sound and Vibration 124 , 329 343,1998.

[18] J.H. He, A coupling method of a homotopy technique and a perturbation technique for non-linear problems, Internat. J. Non-linear Mech. 35, 37-43, 2000.

[19] J.H. He, Homotopy perturbation method: a new nonlinear analytical technique, Appl. Math. compute. 135, 73-79, 2003.

[20] J.K. Zhou, Differential Transformation and its Applications for Electrical Circuits, Huazhong Univ. Pres, Wuhan, China, 1986.

[21] S. Momani, S. Abuasad, Application of He's variational iteration method to Helmholtz equation, Chaos, Solitons Fractals 27, 1119-1123,2006.

[22] Z.M. Odibat, S. Momani, Application of variational iteration method to nonlinear differential equations of fractional order, Internat. J. NonlinearSci. Numer. Simul. 7, 27-34, 2006.

[23] J.H. He, Variational iteration method-a kind of non-linear analytical technique: some examples, Internat. J. Non-linear Mech. 34,699-708,1999. 